Instituto Internacional de Investigación y Desarrollo Tecnológico Educativo INDTEC, C.A.

DOI: https://doi.org/10.29394/Scientific.issn.2542-2987.2020.5.17.11.221-239

OAI-PMH: http://www.indteca.com/ojs/index.php/Revista Scientific/oai

Ensayo Original / Original Essay

\title{
Los desafíos de la inclusión en tiempos de COVID-19
}

\author{
Autores: Carmen Noemí Espinoza Parra \\ Universidad Nacional de Educación, UNAE \\ noemiespinoza58@yahoo.com \\ Azogues, Ecuador \\ https://orcid.org/0000-0002-2223-8648 \\ Gabriela Alexandra Maldonado Regalado \\ Universidad Nacional de Educación, UNAE \\ gabbymaldo@hotmail.com \\ Azogues, Ecuador \\ https://orcid.org/0000-0002-2227-3531 \\ Carlos Julio Rosero Poma \\ Universidad Nacional de Educación, UNAE \\ linespa11@hotmail.com \\ Azogues, Ecuador \\ https://orcid.org/0000-0002-2049-1973
}

\section{Resumen}

La esencia del presente trabajo implica conocer el desafío de la educación inclusiva, ante la inesperada conmoción social que definitivamente afectó al mundo entero, más aún a la población vulnerable del sistema educativo. La interacción de los alumnos con el entorno de aprendizaje afianza conocimientos y fortalece el proceso educativo como proponen Canabal y Margalef (2017). Pero ¿qué sucede en tiempos de COVID-19? El uso de las TIC en el transcurso de este tiempo ha sido de gran importancia, puesto que ha permitido, que la educación se transforme en su totalidad; sin embargo, la brecha digital ha sido el mayor desafío para que los estudiantes obtengan aprendizajes significativos según Gómez, Alvarado, Martínez y Díaz (2018): así pues, la educación inclusiva se ve afectada ya que, las condiciones socioeconómicas y las facilidades tecnológicas no en todos los contextos es igual, de esa manera Torres y Cobo (2017): manifiestan que, la tecnología educativa se encarga del proceso de enseñanza aprendizaje a través del uso de las TIC; sin embargo, frente a estas condiciones en las que actualmente estamos pasando, las tecnologías son concebidas como una barrera más que limita el aprendizaje de los alumnos.

Palabras clave: educación sanitaria; tecnología educacional; aprendizaje.

\section{Cómo citar este ensayo:}

Espinoza, C., Maldonado, G., \& Rosero, C. (2020). Los desafíos de la inclusión en tiempos de COVID-19. Revista Scientific, 5(17), 221-239, e-ISSN: 2542-2987. Recuperado de: https://doi.org/10.29394/Scientific.issn.2542-2987.2020.5.17.11.221-239

Fecha de Recepción: 13-03-2020
Fecha de Aceptación:

05-06-2020
Fecha de Publicación: 05-08-2020 


\title{
The challenges of inclusion in COVID-19 times
}

\begin{abstract}
The essence of this work implies knowing the challenge of inclusive education, in the face of the unexpected social upheaval that definitely affected the entire world, even more so the vulnerable population of the educational system. The interaction of students with the learning environment strengthens knowledge and strengthens the educational process as proposed by Canabal and Margalef (2017). But what happens in the days of COVID-19? The use of ICTs during this time has been of great importance, since it has allowed education to be transformed in its entirety; However, the digital divide has been the greatest challenge for students to obtain significant learning according to Gómez, Alvarado, Martínez and Díaz (2018): thus, inclusive education is affected since, socioeconomic conditions and technological facilities are not the same in all contexts, thus Torres and Cobo (2017): state that educational technology is in charge of the teaching-learning process at through the use of ICT; however, in the face of these conditions in which we are currently experiencing, technologies are conceived as one more barrier that limits student learning.
\end{abstract}

Keywords: health education; educational technology; learning.

How to cite this essay:

Espinoza, C., Maldonado, G., \& Rosero, C. (2020). The challenges of inclusion in CoVID-19 times. Revista Scientific, 5(17), 221-239, e-ISSN: 2542-2987. Recovered from: https://doi.org/10.29394/Scientific.issn.2542-2987.2020.5.17.11.221-239

Date Received: 13-03-2020
Date Acceptance:

05-06-2020
Date Publication:

05-08-2020 


\section{Introducción}

Al hablar del desafío de la educación inclusiva en tiempos de COVID19, es imprescindible abordar el rediseño de la escuela, mismo que implica la adaptación hacia una nueva realidad educativa, iniciando con la implementación de protocolos de bioseguridad, que permitan evidenciar que los alumnos se encuentren en lugares seguros y libres de contaminación; así también, se abordará el replanteamiento de nuevas metodologías y estrategias pedagógicas para mejorar el sistema educativo. Una de ellas es el aula invertida, aquel espacio colaborativo de interacción entre el estudiante y docente.

Sin olvidarnos de la relevancia que conlleva la flexibilización del currículo, la inserción del diseño universal de aprendizaje (DUA), promoviendo de gran manera la educación inclusiva y respondiendo hacia las necesidades de la diversidad; además de los aprendizajes basados en proyectos, los cuales determinan resultados positivos en la escuela con la ayuda de la familia. Además, se analizará el rol del docente descrito por Díez y Sánchez (2015), citados por Espada, Gallego y Gonzalez (2019): ya que "[...] juega un papel importante, puesto que el enfoque inclusivo, implica la implantación de recursos y estrategias que permitan a la comunidad educativa y concretamente al profesorado, afrontar con éxito los cambios que suponen esta nueva practica educativa" (pág. 208).

Finalmente se hará una revisión de la tecnología educativa, que entre muchas de sus bondades está el uso de las TIC, se realizará un breve análisis de cómo influye en estos momentos de conmoción social, no así también la influencia de la Web 2.0, dentro de la educación inclusiva y de la brecha digital misma que precisamente ahora, compone un elemento fundamental dentro de distintos contextos en los que muchos alumnos se hallan inmersos. El futuro éxito de la educación inclusiva dependerá, por lo tanto, del planteamiento de estas barreras como un desafío para la sociedad. 


\section{Desarrollo}

El avance de los procesos de la educación inclusiva en la escuela del siglo XXI, se truncó por una barrera casi invisible, el COVID-19. Ahora, debemos analizar cuáles son los desafíos que la inclusión tiene que superar en un contexto post pandemia.

\subsection{Rediseño de la escuela}

El objetivo de una escuela durante el confinamiento cambió y ahora debe adaptarse al nuevo hábitat en camino hacia su reapertura. Para reabrir las instituciones educativas, hay que tomar en cuenta las características de la escuela inclusiva, descritas por la UNESCO (1994), citada por la Organización de las Naciones Unidas para la Educación, la Ciencia y la Cultura (UNESCO, 2004):

[...] Deben reconocer las diferentes necesidades de sus alumnos y responder a ellas, adaptarse a los diferentes estilos y ritmos de aprendizaje de los niños y garantizar una enseñanza de calidad a través de un programa de estudios apropiado, una buena organización escolar, una utilización adecuada de los recursos y una relación con sus comunidades [...] (pág. 20).

Observando los requerimientos planteados y a tenor de las medidas de distanciamiento social que impongan los gobiernos, lo fundamental al retornar a la escuela inclusiva será: encontrarse, ser reconocido y valorado por otros, y atender a la nueva diversidad de necesidades educativas provocadas por el entorno del COVID-19.

La sociedad sobrevaloró el alcance digital que habíamos logrado en educación. Ningún sistema educativo estuvo capacitado para simular la educación presencial de una forma virtual. La pedagogía de la presencia es la que realmente otorga justicia social, porque compensa las diferencias de origen, a través de las distintas interacciones profesor-estudiante. 
Afirman Canabal y Margalef (2017), que los docentes: "valoramos el papel fundamental que juega la inclusión de los componentes afectivos así como la atención a qué cualidades, habilidades y actitudes se movilizan; cómo se abordan los procesos de aprendizaje; en definitiva, qué competencias se ponen en juego" (pág. 167).

Es evidente, que no son necesarios grandes cambios arquitectónicos en las escuelas inclusivas, salvo pequeños planes de contingencia y bioseguridad para una apertura con alternativas como: jornadas diversas, horarios de ingresos escalonados, asistencia intercaladas por semanas; lo más importante es que se abra lo más pronto posible porque la presencia de los actores educativos en la escuela es insustituible.

\subsection{Redes de apoyo para la inclusión}

La pandemia, puso de manifiesto que el trabajo de la inclusión es una tarea de toda la sociedad y no solamente de la escuela, a la cual se le venía exigiendo de manera implícita o explícitamente. Por el contrario, el apoyo comunitario debe obedecer en gran medida a las conciliaciones que se consigan entre las redes de apoyo.

\subsubsection{Red de personas naturales}

En la inclusión, manifiestan Angenscheidt y Navarrete (2017): que "el docente puede ser un facilitador o una barrera hacia el mismo. Su actitud hacia la implementación de prácticas educativas inclusivas puede promover la participación plena y efectiva del alumnado o puede minimizarla" (pág. 240).

El confinamiento expuso las debilidades dentro de la virtualidad de los docentes. En los últimos años, las capacidades digitales de profesores y sofisticación tecnológica de las instituciones educativas han ido incrementándose de manera desigual. La mayoría ha mejorado mucho, pero no al ritmo y calidad deseada, pero en ningún caso hubo una preparación 
adecuada en herramientas tecnológicas virtuales que atiendan necesidades educativas especiales.

En la educación de niños con o sin discapacidad, la familia es una red natural de apoyo fundamental. Acorde a lo que concluyen Llamazares, Arias y Melcon (2017a): "los padres han de tomar conciencia de que poder interactuar en las tareas, ejercicios y actividades con sus hijos con discapacidad implica un nivel de acercamiento que difícilmente se logra dar en el hogar" (pág. 207). En estos momentos la educación presencial está vetada por los requerimientos sanitarios. La familia pudo verse sobrepasada por el aislamiento en el hogar, llegándose a comprobar que no es el lugar físico ni pedagógico adecuado para que funcione la escuela. La inclusión de los niños con necesidades educativas ha sufrido una paralización, por la falta de preparación de sus padres.

La formación de nuevas habilidades y competencias tecnológicas, basadas en la ética y el liderazgo en ambientes virtuales, se deben promover con un desarrollo profesional docente. También, valorar la capacidad que tiene la familia para trabajar en competencias emocionales son opciones valederas para afrontar estos retos.

\subsubsection{Red de instituciones especializadas}

La justicia y equidad social que propugnan las instituciones dedicadas a evitar la vulnerabilidad educativa de niños con necesidades educativas especiales, se apoyan en el trabajo individualizado descrito por Llamazares, Arias y Melcon (2017b): “[...] los profesionales educativos, psicólogos, especialistas en la discapacidad, que ayudan y asesoran a los padres en la formación de los hijos, su formación tiene que ir a la par, del ámbito escolar al ámbito del hogar [...]" (pág. 207). La crisis sanitaria, está provocando una dificultad económica, que en algunos países ha obligado a reducir el gasto público en educación a pesar de que hacerlo es tocar a los más vulnerables y 
las instituciones especializadas han sido las más afectadas.

La educación es una inversión no un gasto. La carta de presentación de un país para el mundo es la educación, como base sustentable para el desarrollo presente y futuro de su sociedad; por lo tanto, para salir de la crisis social hay que ser anti-modelo, es decir invertir más en educación, investigación, emprendimiento e innovación. El camino de la inclusión dependerá de factores exógenos como el económico y decisiones políticas adversas, estas serán las mayores luchas que deberá afrontar ahora.

\subsection{Un nuevo modelo educativo}

El nuevo escenario en cada centro educativo será distinto por los planes de contingencia que obligan a respetar una distancia de seguridad entre las personas, provocando que cada aula albergue la mitad de su capacidad física. Ofrecer un tipo de educación mixto entre un modelo presencial y virtual demandará más recursos humanos y técnicos, de parte de los Ministerios de Educación y cambios estructurales dentro de los contenidos de los planes educativos.

\subsubsection{El currículum}

Regresar a la antigua normalidad, sería retornar a un currículo muy bibliográfico y abundante de contenidos redundantes. Surge la necesidad de presentar nuevos modelos educativos que formen competencias con destrezas esenciales no centradas en asignaturas, sino en ámbitos y en aprendizajes basados en proyectos. Así lo da a conocer Bolivar (2019):

Frente a los discursos de integración de la diversidad y a las estrategias de las necesidades educativas especiales, centradas en las intervenciones en el aula, abogamos actualmente por una perspectiva de la escuela como conjunto donde tiene sentido un currículum inclusivo, al tiempo que obviamente- se interviene en el contexto sociocultural y político más amplio. Una inclusión no puede florecer sino en el seno de 
una cultura escolar favorable, por lo que las acciones didácticas un aula particular, aun siendo relevantes, por sí solas, poco lejos pueden ir. Expandir la visión del aula a la escuela como conjunto es un paso necesario (pág. 848).

Flexibilizar el currículo facilitaría una enseñanza que ayude a potenciar el talento de cada alumno. Esto afectaría al sistema tradicional de evaluación cuantitativa, cuando se trata de calificar a los alumnos; pero son los mismos que en este confinamiento se han visto obligados a seguir formándose en remoto con adaptaciones a los contenidos de la materia en búsqueda de los aprendizajes esenciales. Este nuevo diseño curricular inclusivo deberá evaluar si el alumno ha superado de manera cualitativa los objetivos generales del currículo y ha desarrollado las competencias y destrezas que le permiten promocionar al siguiente curso.

\subsubsection{Estrategias pedagógicas}

Las estrategias pedagógicas utilizadas durante las clases virtuales no alcanzaron a estudiantes inmersos en educación inclusiva, son aspectos de análisis profundo por los investigadores educativos en tiempos de pandemia, algunas necesitarán ser repotenciadas en el retorno a las aulas.

\subsubsection{Aula invertida}

El modelo mixto que parta desde una educación presencial y virtual, se consideraría el más aceptado por los países para el regreso a las aulas. Este nuevo enfoque educativo se apoyaría en la taxonomía de Bloom, representante de la estrategia de aulas invertidas, reflejada en la figura 1. 
Figura 1. Taxonomía de Bloom y la clase invertida (Adaptación y traducción de la imagen original).

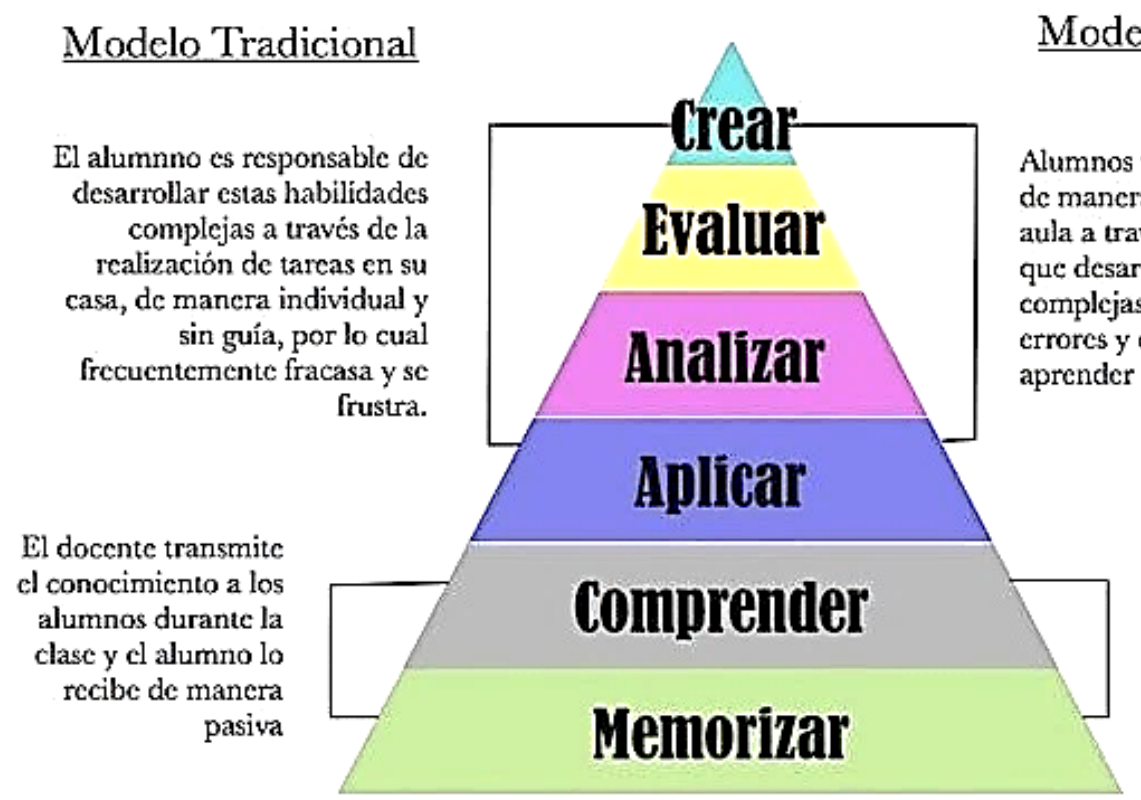

El alumno adquicre el contenido de la clase en casa a través de videos que puede manipular para aprender a su propio ritmo

Fuente: Williams (2013); traducida por los Autores (2020).

Las plataformas virtuales y el uso de nuevas tecnologías para Hernández y Tecpan (2017a): dentro de un "modelo de aula invertida puede facilitar el acceso a contenidos seleccionados por el docente, para su uso antes, durante y después de clases, o como espacio colaborativo para facilitar la interacción entre estudiantes y profesores fuera de la clase" (pág. 195). Y cómo enfatizan Platero, Tejeiro y Reis (2015), citados por Hernández y Tecpan (2017b):

El aula invertida como metodología y herramienta de aprendizaje, invierte el modo de enseñanza tradicional provocando que gran parte de la obtención de conocimiento se produzca mediante el aprendizaje autónomo con el estudio fuera del aula y que la realización de tareas y prácticas se hagan en el aula con el apoyo de los compañeros y el docente, transfiriendo el control del aprendizaje al estudiante [...] (pág. 195). 
Invertir el aprendizaje, es una de las estrategias pedagógicas valederas que más se adapta al modelo virtual y presencial propuesto; aprovecha al máximo el potencial de las plataformas virtuales y TIC, apoyándose de las interacciones propias del aula presencial.

\subsubsection{Diseño Universal de Aprendizajes (DUA)}

Las dificultades de aprendizaje provocadas por el entorno de aislamiento sufrido, se deben asumir desde la integración de una flexibilidad en la planificación y desarrollo curricular propuesto por Valencia (2017): "el currículo educativo tradicional es diseñado para responder a las necesidades del alumno promedio, excluyendo a aquellos que presentan alguna discapacidad, se encuentran en otras situaciones de desventajas o presentan diferentes habilidades, estilos de aprendizaje y preferencias [...]" (pág. 108).

La presencia obligatoria de los estudiantes en clase era requerida en el modelo educativo antes del COVID-19, se ajustaba un currículo universal que buscaba aprendizajes mínimos estandarizados y con adaptaciones dirigidas a los estudiantes con discapacidades. Al diversificar ahora la oferta educativa se necesita flexibilizar contenidos, objetivos, evaluación; es decir la implantación del DUA como estrategia pedagógica permite atender a la diversidad del aula desde un comportamiento humano distinto y con compromiso con la sociedad.

\subsubsection{Aprendizaje Basado en Proyectos (ABP)}

La estrategia ABP plantea desafíos a la inclusión por la virtualidad de los posibles trabajos que se deberán planificar fuera del aula. La elaboración de proyectos tiene ventajas, en este sentido para Reverte, Gallego, Molina y Satorre (2006), citados por Fernández-Cabezas (2017): "el uso de esta metodología reporta al alumnado más satisfacción y aumenta su interés, no resultándoles difícil llevarla a cabo. Los alumnos valoraron positivamente el relacionar los contenidos teóricos con su futuro profesional, aplicándolos a la 
realidad" (pág. 276).

Los trabajos colaborativos basados en Aprendizajes Basados en Proyectos (ABP) pueden conseguir resultados positivos en la inclusión, si la elaboración de los mismos se ofrece en una forma organizada dentro y fuera del aula con el apoyo de la familia.

\subsection{La tecnología educativa}

Mencionando a Torres y Cobo (2017), definen a la tecnología educativa como: "[...] aquellos recursos relacionados con los medios de comunicación (cine, televisión, radio, internet) que sirven y son responsables para transmitir contenidos con valor educativo a un grupo de participantes o una sociedad" (pág. 33). Dicha tecnología aporta a la educación herramientas valiosas que facilitan procesos educativos y que por sus condiciones se oferta de forma holística a sus usuarios.

El cambio trascendental que tuvo la educación en tiempos de COVID19 ha puesto en manifiesto aspectos de desigualdad dejando una marca visible en niños con necesidades educativas especiales, condiciones económicas bajas, ubicación geográfica, etc., y estudiantes que por lo contario gozan de recursos y medios para una educación virtual.

\subsubsection{Evolución}

La tecnología en los últimos años ha crecido a pasos agigantados. En el contexto educativo se ha hecho presente aportando a la educación herramientas que le permiten un adecuado desarrollo en procesos de enseñanza aprendizaje.

Interpretando a Correa y Pablos (2009): "la introducción de las TICs en el contexto educativo ha dado un nuevo impulso a la pedagogía, estimulando al sistema escolar en la búsqueda de nuevos caminos para aprender" (pág. 134). Bajo este enfoque, Heras, Orden y Serrano (2020): aseveran que la 
implementación de las "[...] Tecnologías en la Educación Inclusiva, se ha convertido en un pilar fundamental en nuestros días, facilitando el acceso a la información y comunicación, potenciando en los estudiantes [...]" (pág. 338).

Los recursos tecnológicos han estado a disposición de los usuarios, sin embargo, no han sido utilizados y aplicados en su totalidad, esto ha generado que muchas personas al enfrentar la realidad que hoy acoge al mundo evidencien una falta de preparación para afrontar el reto de la virtualidad.

Actualmente los salones y clases presenciales han dado un giro total, migrando hacia el hogar con una modalidad distinta en donde la tecnología y la virtualidad ya no es una opción sino una necesidad obligatoria para cumplir con la educación.

\subsubsection{La brecha digital}

La situación que vive el Ecuador y el mundo ha hecho que se modifique la educación en su manera de enseñanza aprendizaje, esta trasformación permite dar un vistazo a las fallas en materia de equidad que enfrenta el sistema educativo.

En este sentido, Gómez, Alvarado, Martínez y Díaz (2018): expresan que la brecha digital se refiere a la inequidad entre aquellos que tienen o no acceso físico a la TICs. Es un concepto claro de la desigualdad o brecha que existe en contextos sociales y educativos diferenciando entre ricos y pobres; entre educación pública y privada y entre estudiantes normales y con necesidades educativas especiales.

La educación antes de pandemia se desarrollaba en un sistema inclusivo aceptando a la diversidad y fomentando la igualdad y equidad de derechos; actualmente considerando las situaciones reales que experimenta la educación y sociedad ya no se puede hablar de una educación inclusiva puesto a que la exclusión y desventaja que viven muchos estudiantes impiden no solamente el acceso a la tecnólogo y virtualidad sino también a la 
educación.

\subsubsection{Web 2.0}

Frente a la situación resulta propicio indagar y emplear plataformas virtuales que ofrece la web, sobre todo considerando la inclusión educativa lo que permite incursionar sobre estrategias y herramientas que brinda la web a base de una conectividad. Esto no únicamente permite procesos de enseñanza aprendizaje sino además niveles de comunicación entre sus usuarios.

Hoy la web 2.0 en el proceso de enseñanza-aprendizaje, ha significado para Martínez (2010): "la participación activa de los alumnos/usuarios, con espíritu colaborativo, en la construcción social de conocimiento" (pág. 180); por lo tanto, permite la web 2.0 conectar las nuevas habilidades digitales de los educandos con un modelo de aprendizaje más participativo e innovador.

\section{Conclusiones}

La educación inclusiva en tiempos de pandemia es una realidad, que acoge a nivel mundial una situación que ha desestabilizado a la sociedad y consigo a la educación.

Se hablaba sobre una era digital, del uso de las tecnologías y virtualidad aplicada en diversos ámbitos uno de ellos la educación; pero, como un proceso lejano que en algún momento sería aplicado. Actualmente está claro que la virtualidad no estuvo distante de ser aplicada y utilizada diariamente por casi una totalidad de usuarios, aunque es evidente también que como sociedad no estábamos preparados para afrontar este nuevo sistema que nos acoge.

Los docentes nos hemos visto obligados a cambiar nuestra metodología, sistemas de enseñanza y labor presencial emigrando a una virtualidad, utilizando herramientas tecnológicas y de comunicación para llegar a los estudiantes brindando apoyo e información acorde al currículo. 
En este sentido, los estudiantes y sus familias frente a este confinamiento y el nuevo sistema de educación han tenido que modificar y reestructurar sus estilos de vida a más de ello buscar de manera autónoma preparación ante la virtualidad para aplicar a su realidad y brindar la ayuda que el alumno necesita.

Por otro lado, se amplió la brecha social y educativa existente, en donde los más afectados son cuyas personas se encuentran en niveles económicos bajos, ubicación geográfica desfavorable, falta de manejo o desconocimiento de las tecnologías, niños/as y adolescentes con necesidades educativas especiales y personas o familias diagnosticas con COVID-19.

Está claro que no todo el contexto tiene una misma realidad, docentes, estudiantes y una sociedad en si enfrentan situaciones diferentes, por un lado, están los privilegiados de contar con la tecnología necesaria para trabajar y estudiar; y por otro, los vulnerables pudiendo ser considerados incluso como excluidos.

La educación presencial es insustituible, esta es una realidad que enfrenta toda una sociedad, sin embargo, no podemos desmayar frente a la situación por el contrario demostrar resiliencia y una capacidad de adaptación a un nuevo sistema de educación y diario vivir.

\section{Referencias}

Angenscheidt, L., \& Navarrete, I. (2017). Actitudes de los docentes acerca de la educación inclusiva. Ciencias Psicológicas, 11(2), 233-243, eISSN: 1688-4221. Recuperado de:

https://doi.org/10.22235/cp.v11i2.1500

Bolivar, A. (2019). Un currículum inclusivo en una escuela que asegure el éxito para todos. e-Curriculum, 17(3), 827-851, e-ISSN: 1809-3876. Recuperado de: https://doi.org/10.23925/1809-3876.2019v17i3p827- 
Canabal, C., \& Margalef, L. (2017). La retroalimentación: la clave para una evaluación orientada al aprendizaje. Profesorado: Revista de Currículum y Formación de Profesorado, 21(2), 149-170, e-ISSN: 1138414X. Recuperado de:

https://www.redalyc.org/articulo.oa?id=56752038009

Correa, J., \& Pablos, J. (2009). Nuevas tecnologías e innovación educativa. Revista de Psicodidáctica, 14(1), 133-145, e-ISSN: 1136-1034. Recuperado de: https://www.redalyc.org/articulo.oa?id=17512723009

Espada, R., Gallego, M., \& Gonzalez, R. (2019). Diseño Universal del Aprendizaje e Inclusión en la Educación Básica en Ecuador. Alteridad, 14(2), 207-218, e-ISSN: 1390-8642. Recuperado de: https://doi.org/10.17163/alt.v14n2.2019.05

Fernández-Cabezas, M. (2017). Aprendizaje basado en proyectos en el ámbito universitario: Una experiencia de innovación metodológica en educación. INFAD, 2(1), 269-278, ISSN: 0214-9877, e-ISSN: 26035987. Recuperado de: https://doi.org/10.17060/ijodaep.2017.n1.v2.939 Gómez, D., Alvarado, R., Martínez, M., \& Díaz, C. (2018). La brecha digital: una revisión conceptual y aportaciones metodológicas para su estudio en México. Entreciencias, 6(16), 49-64, e-ISSN: 2007-8064. Recuperado de:

https://doi.org/10.22201/enesl.20078064e.2018.16.62611

Heras, M., Orden, R., \& Serrano, V. (2020). Las tecnologías en la organización de un aula inclusiva para niños con capacidades especiales. Revista Scientific, 5(16), 334-351, e-ISSN: 2542-2987. Recuperado de: https://doi.org/10.29394/Scientific.issn.25422987.2020.5.16.18.334-351

Hernández, C., \& Tecpan, S. (2017a,b). Aula invertida mediada por el uso de plataformas virtuales: un estudio de caso en la formación de profesores de física. Estudios Pedagógicos, 43(3), 193-204, e-ISSN: 
0718-0705. Recuperado de: https://doi.org/10.4067/S0718$\underline{07052017000300011}$

Llamazares, J., Arias, A., \& Melcon, M. (2017a,b). El papel de los profesionales y la familia ante la diversidad funcional desde la revisión teórica. HOLOS, 7, 198-212, e-ISSN: 1807-1600. Recuperado de: https://doi.org/10.15628/holos.2017.5592

Martínez, F. (2010). Herramientas de la Web 2.0 para el aprendizaje 2.0. Revista de Artes y Humanidades UNICA, 11(3), 174-190, e-ISSN: 1317102X. Recuperado de:

http://www.redalyc.org/articulo.oa?id=170121969008

Torres, P., \& Cobo, J. (2017). Tecnología educativa y su papel en el logro de los fines de la educación. Educere, 21(68), 31-40, e-ISSN: 13164910. Recuperado de:

https://www.redalyc. org/articulo.oa?id=35652744004

UNESCO (2004). Temario abierto sobre educación inclusiva: Materiales de Apoyo para Responsables de Políticas Educativas. ISBN: 9568302-15-8. Santiago, Chile: Oficina Regional de Educación de la UNESCO para América Latina y el Caribe OREALC / UNESCO.

Valencia, C., Hernández, O. (2017). El Diseño Universal para el Aprendizaje, una alternativa para la inclusión educativa en Chile. Atenas, 4(40), 105-120, e-ISSN: 1682-2749. Recuperado de: https://www.redalyc.org/articulo.oa?id=478055150008

Williams, B. (2013). How I flipped my classroom. Norfolk, Nebraska: NNNC Conference. Recovered from:

https://nextgenerationextension.org/2013/10/01/blooms-and-theflipped-classroom/ 


\section{Ensayo Original / Original Essay}

\section{Carmen Noemí Espinoza Parra}

e-mail: noemiespinoza58@yahoo.com

Nacida en Cañar, provincia del Cañar, Ecuador, el 5 de

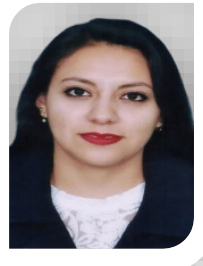
agosto del año 1994. Los estudios primarios los realicé en la escuela Alfonso María Ortiz y el bachillerato en el colegio José Peralta obteniendo el título de Químico Biólogo; mis estudios de pregrado los realice en la Universidad Católica de Cuenca (UCACUE), obteniendo el título de Licenciada en Ciencias de la Educación mención Sicología Educativa y Orientación Vocacional en el año 2017; Desempeño funciones como Docente en la Unidad Educativa Particular Universitaria de Azogues, ubicada en el campus de la Universidad Católica de Cuenca (UCACUE), sede Azogues desde el año 2018 hasta la actualidad. 


\section{Ensayo Original / Original Essay}

\section{Gabriela Alexandra Maldonado Regalado \\ e-mail: gabbymaldo@hotmail.com}

Nacida en Azogues, provincia del Cañar, Ecuador, el 30

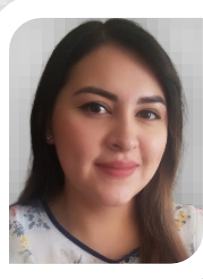
de junio del año 1993. Los estudios primarios los realice en la escuela Rafael María García, mientras que el bachillerato en el colegio Luis Cordero, obteniendo el título de Químico Biólogo en el bachillerato internacional; los estudios de pregrado los realice en la Universidad Católica de Cuenca (UCACUE), obteniendo el título de Licenciada en Ciencias de la Educación mención Educación Inicial y Parvularia en el año 2018; desempeño el cargo de Directora en el Centro de Educación Inicial Super Babies GYM de la ciudad de Azogues, desde el año 2018 hasta la actualidad. 


\section{Carlos Julio Rosero Poma}

e-mail: linespa11@hotmail.com

Nacido en Zamora, provincia de Zamora Chinchipe,

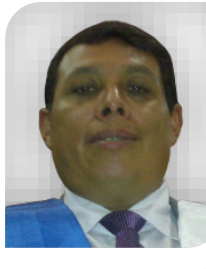

Ecuador, el 19 de noviembre del año 1971. Con estudios primarios en la escuela Cristóbal Colón en la ciudad de Tulcán y el bachillerato en Ciencias Sociales en el Colegio la Dolorosa en Loja; realicé estudios de pregrado en la Universidad Técnica Particular de Loja (UTPL), obteniendo el título Licenciado en Ciencias de la Educación mención Informática en el año 2012; Desempeño funciones como Docente de Educación para la Ciudadanía, de la Unidad Educativa "Daniel Álvarez Burneo" de la ciudad de Loja desde el año 2016 hasta la actualidad. 